\title{
'No tenemos que enseñárselo todo a los profesores'. Los derechos de acceso a la ciberrealidad y sus riesgos desde la mirada de niños y niñas
}

'We do not have to show everything to teachers'. The right to access to cyberreality and its risks from the outlook of children.

\section{Iván Rodríguez-Pascual}

Profesor de Sociología. Facultad de Trabajo Social. Universidad de Huelva. España ivan@uhu.es

\author{
Elena Morales-Marente \\ Profesora de Psicología Social. Facultad de Ciencias de la Educación, Universidad de Huelva. España. \\ elena.morales@dpsi.uhu.es
}

\section{Estrella Gualda \\ Profesora Titular de Sociología, Grupo de Investigación Estudios Sociales e Intervención Social. Universidad de Huelva. España. estrella@uhu.es}

\section{RESUMEN}

Este artículo presenta la percepción que niñas y niños andaluces tienen de sí mismos, sus derechos y su relación con el profesorado y el resto de la población adulta en el contexto de la sociedad de la información, particularmente, respecto al discurso sobre los riesgos de existentes en esta nueva (ciber)realidad. Basados en datos cuantitativos y cualitativos, tras la realización de una encuesta y una serie de entrevistas grupales en Andalucía, los resultados apuntan hacia una visión compleja del riesgo digital y su afrontamiento por parte de la población infantil, así como hacia la demanda de un rol o posición ambivalente a este respecto por parte de las personas adultas.

\section{ABSTRACT}

This study presents how Andalusian children perceive themselves, their rights, and their relationships with teachers and the adult population in the context of information society, especially when the matter under discussion is the risk related to cyberreality. Based on the combination of quantitative (survey) and qualitative (group interview) methodologies, the results showed a complex representation of risk and the way to manage it by children and their peers. The results also show an ambivalent position reserved for adults in this discourse: they are both a source of protection and, at the same time, a potential risk for children's rights, especially the right to privacy.

PALABRAS CLAVE

sociedad de la información | cibercultura | riesgo digital | infancia | derechos digitales y comunicación KEYWORDS

information society | cyberculture | digital risk | childhood | digital rights and communication

\section{Introducción (1)}

La irrupción del nuevo escenario digital, poblado por generaciones a las que etiquetamos siguiendo la terminología de Prensky (2001) como nativos digitales, ha añadido nuevas incógnitas en torno a la manera en que las personas adultas y niños conviven y hacen uso de estos nuevos medios de comunicación. Como bien señala el profesor Buckingham (2000) en un libro que es ya un clásico contemporáneo y que titula con acierto La muerte de la Infancia (2): crecer en la era de los medios electrónicos, con demasiada frecuencia nuestro discurso ha resultado polarizado entre las posiciones acríticas y tecnoutópicas, que atribuyen a las nuevas tecnologías de la comunicación por sí mismas cierto potencial revolucionario, y las que giran en torno al enfoque defensivo que remite a cualquier tiempo pretecnológico como un edén donde la infancia y los adultos convivían a salvo de blogs, chats y redes sociales. Este cierto desencuentro convierte a los niños y niñas en el discurso adulto en un objeto nombrado y argumentado, que solo raramente actúa y representa sus propios intereses; su ciudadanía es sólo potencial así como son posibles víctimas necesitadas de 
protección, lo que los convierte sistemáticamente en objetos del debate generado al calor del pánico moral o la instrumentalización dramática -y discursiva- de su condición infantil (Dean 1999, Kuipers 2006, Hodson 2010, Selwyn 2003 y Wold 2010). Este discurso tiende a ser adultocéntrico (porque toma el criterio adulto en un sentido normativo) y atribuye al niño rasgos y motivaciones que con frecuencia es difícil encontrar en el discurso infantil (Rodríguez-Pascual 2010b). Es difícil, por tanto, encontrar en él una representación que describa a niños y niñas desde la perspectiva de sus propios intereses, en tanto individuo y sujeto de derechos.

Paralelamente, el nuevo mundo de la comunicación digital cada vez más tiene un rostro más joven y en el contexto de la Unión Europea la edad de los usuarios de Internet desciende progresivamente (3). Algunos autores incluso afirman que asistimos a un giro copernicano que nos lleva desde un mundo de cultura sólida a otro de información "líquida" que convierte la nueva alfabetización digital en un derecho de los individuos y una condición para una nueva democratización (Area y Pessoa 2012); un mundo en el que el paisaje mediático se hace más complejo y dependiente del contexto y la posición social de sus actores, de tal forma que no existe ningún impacto "natural" de los nuevos medios en la vida infantil, sino una apropiación (ciber)cultural y compleja de estos (Cabrera 2013, De Almeida y otros 2011, Hirsjävi y Tayie 2011). Un mundo, en fin, en el que no descartamos que junto a una clara ampliación y globalización de las maneras de comunicar (y comunicarse) en el entorno de la llamada cultura digital estén surgiendo también formas menos comprometidas y/o veraces en la relación interpersonal de los más jóvenes (Colorado 2010, García y Rosado 2012).

En este texto damos cuenta de la manera en que estos sujetos del escenario digital se ven a sí mismos y a la población adulta, particularmente, desde el ámbito de los derechos personales y su relación con los nuevos medios. Nuestros instrumentos de investigación, al dirigirse a los niños y niñas como informantes, descubren un sujeto cualificado que produce discursos complejos y ricos en torno tanto a su relación con los nuevos medios, como con las personas adultas que les acompañan, muy especialmente padres, madres y docentes. La investigación reciente (Vergara y Vergara 2012) encuentra una compleja noción relacional de la infancia que ve el control adulto como una amenaza a la libertad individual y contempla ya nuevos valores, entre los que se encuentran los derechos humanos, y dentro de estos, los derechos de los niños. ¿Cómo conjugar entonces una presencia creciente de usuarios menores de edad con la construcción de un marco de uso seguro y productivo, sin subestimar su capacidad ni renunciar a respetar de manera integral sus derechos, no sólo exclusivamente en términos de protección frente al riesgo?

En nuestra opinión la clave de la cuestión radica en la extrema ambivalencia en que nos movemos. Tengamos en cuenta que la propia Convención de Derechos del Niño (CDN) (Naciones Unidas 1989) tiende a conjugar tanto la protección del niño frente a toda forma de violencia y explotación (artículos 19 y 36) como el derecho a la libertad de expresión y acceso a la información o el respeto por su privacidad (artículos 13 y 16) así como el de la participación social (artículo 31), por poner sólo algunos ejemplos. Resulta difícil no encontrar contextos y situaciones en los que algunos de estos derechos no parezcan contradecirse, necesitando de una articulación compleja. Demasiada protección puede asfixiar a los menores de edad como sujetos y convertirlos en excluidos de facto de la sociedad de la información, mientras que demasiado poca puede producir entornos de riesgo inaceptables (Ruxton 2006). Hasta ahora esta aparente contradicción se ha resuelto bien del lado de los intereses adultos, bien incorporando al niño de manera preferente desde la visión de las necesidades evolutivas o como sujeto de protección, subordinado a esos mismos intereses adultos. En su estudio comparativo entre la legislación brasileña y europea, por ejemplo, la profesora Amich-Elias (2007) pone de manifiesto cómo los términos protección de la infancia y libertad de expresión suelen aparecer como antagonistas en el debate legal, si bien deberían ser aspectos complementarios de una visión integral de derechos de la infancia. Otras investigaciones centradas en el marco de la Unión Europea vienen a destacar igualmente la falta de articulación entre diferentes ámbitos de regulación de este complejo conjunto de derechos en el escenario de la comunicación digital desde la visión del menor de edad como sujeto de derechos (Garde 2011 y 2012 , Rodríguez-Pascual 2010a y Savirimuthu 2011). O’Neill (2010) añade que la raíz del problema es la consideración de la relación con estas nuevas tecnologías como un problema estrictamente individual que debe ser negociado y articulado por cada individuo; en su lugar propone construir un debate sobre derechos de la comunicación que descanse sobre una perspectiva de derechos aplicada al desarrollo de una nueva alfabetización digital.

En nuestra opinión, parte del problema radica en que no se toma con suficiente frecuencia a niños y niñas como informantes sobre estos aspectos. El proyecto "Infancia 2.0: redes sociales y usos tecnológicos 
emergentes en la población nativa digital andaluza", del cual ofrecemos detalle metodológico en el apartado siguiente, ha constituido un intento de reconstruir, desde la mirada de la propia infancia, la representación relacional que les vincula a las personas adultas a través del discurso sobre el riesgo y oportunidades del mundo digital y sus derechos.

Nos gustaría en este artículo responder no sólo a la pregunta, por otro lado bastante obvia de ¿cómo nos ven?, sino también a "cómo se ven". Es decir, hemos intentando que no sólo nos retraten como adultos sino que se retraten a sí mismos en tanto usuarios complejos de estas nuevas tecnologías de la comunicación. En este sentido, cabe destacar como principales objetivos de este texto:

1. Conocer la actitud de niños y niñas frente a los riesgos del nuevo escenario de la comunicación digital.

2. Describir, contando con los niños y niñas como informantes, el papel que desempeñan los adultos en relación a estos riesgos y también a los derechos de los niños y niñas.

3. Finalmente, proporcionar una descripción fidedigna de la manera en que niños y niñas perciben sus derechos como individuos en los nuevos entornos digitales y la relación de ésta con dos variables clave: la edad y el género (4).

\section{Métodos}

Este texto es el fruto de una explotación parcial de los datos recogidos durante el desarrollo del proyecto Infancia 2.0. Una parte importante del proyecto se dedicó al estudio de la percepción que revelaban los niños y niñas andaluces (5) entre los 11 y los 18 años en torno a cuestiones claves como el riesgo, la comunicación y la participación social a través de las nuevas tecnologías o sus derechos en un escenario digital. Al mismo tiempo, se apostó tanto por la integración de técnicas cuantitativas y cualitativas como, en el trabajo de campo, por una gestión respetuosa de la relación entre investigadores y sujetos investigados y en la que los niños y niñas no se sintieran forzados a participar.

Para lograr un acceso diversificado a las distintas opiniones y circunstancias vitales de parte de este colectivo, el proyecto ha trabajado con población escolarizada entre los 11 y 18 años en más de una decena de centros públicos de la red educativa andaluza a lo largo de un trabajo de campo que comenzó con 2011 y no acabó hasta junio de ese mismo año. La siguiente ficha técnica expone someramente las claves metodológicas del proceso con el que se han producido estos datos.

\section{Ficha Técnica}

Metodología: cuantitativa y cualitativa (cuestionario cerrado y entrevista grupal).

Universo: población andaluza escolarizada en centros de la red pública de enseñanza entre 11 y 18 años.

Muestra: polietápica. 784 casos distribuidos en cinco de las ocho provincias andaluzas. Ponderación final por sexo, edad y tamaño de hábitat.

Nivel de confianza: $95 \%$

Error muestral: $p=q=0,5$ de $\pm 4 \%$

Como puede apreciarse, el tamaño muestral conseguido durante la aplicación del instrumento cuantitativo (cuestionario) es significativamente alto. Cabe destacar que el horizonte geográfico y socioeconómico de la muestra es también amplio, en la medida en que la distribución de los puntos de muestreo empleados para extraer a cada uno de estos individuos abarca tanto un conjunto muy diverso de centros educativos esparcidos por diferentes hábitats (desde capitales de provincia al entorno rural disperso), un área geográfica muy amplia, quedando representada la región andaluza. Se trata, por tanto, de una muestra grande y representativa del ámbito poblacional diverso del que se extrae.

lgualmente interesante para nuestros propósitos resulta la cuestión del tipo de instrumento de observación utilizado en esta investigación, así como los criterios de su aplicación. Dicho instrumento constaba de un 
cuestionario adaptado con 28 preguntas redactadas de una manera amigable y clara (una muestra se incluye en el anexo I). Se decidió su aplicación autoadministrada dentro del grupo de aula. Durante su aplicación estaban presentes tanto el/la profesor/a a cargo del grupo como el/la investigador/a encargado del trabajo de campo, si bien al primero se le pedía que no interviniera y el segundo se limitaba a presentar la investigador y a explicar algunos aspectos básicos de la misma (libre participación, confidencialidad. etc.), para después repartir los cuestionarios entre los estudiantes y resolver puntualmente las dudas que surgieran en la respuesta autoadministrada del mismo. Con posterioridad a este trabajo de campo, y con ayuda del software estadístico SPSSPC 15.0, se digitaron y depuraron los datos contenidos en los cuestionarios.

Asumimos también, al partir de este diseño de investigación, algunas limitaciones: la primera y más obvia es la inevitable cosificación que el lenguaje pautado y objetivado propuesto por los cuestionarios ofrece para tratar un tema complejo y lleno de matices, que al final busca retratar la identidad que niños y niñas construyen a diario en un entorno virtual. La presencia del discurso cualitativo ayudaba a minimizar este efecto, facilitando una posible triangulación. Por otro lado, es igualmente cierto que el sistema de aplicación del cuestionario ha sido en contexto grupal y no ha contado con una completa privacidad para los informantes en la medida que, habitualmente, sus docentes se encontraban presentes en el aula. Hemos tratado de minimizar esta distorsión en el primer caso ofreciendo unas instrucciones muy minuciosas para su cumplimentación y apoyo presencial por parte de los investigadores durante el trabajo de campo, explicando también a profesores y profesoras, en el segundo caso, la necesidad de que no intervinieran durante la sesión y dejaran que fuera el personal investigador el que pasara a primer plano. La consistencia y coherencia de los datos que aquí ofrecemos nos hacen pensar que estas limitaciones no han tenido un efecto reseñable en la investigación.

Dado que el estudio partió con voluntad multimetodológica contamos, paralelamente a estos más de 780 casos observados, con seis entrevistas grupales realizadas en diferentes centros educativos de la red pública andaluza que han sido convenientemente transcritas y analizadas, de las que en este mismo texto se ofrecen algunos extractos (6) y que han incorporado en su diseño el uso de técnicas de focalización del discurso a través de cuentos inconclusos o de descripción de situaciones que los niños y niñas debían analizar. En realidad, las distintas técnicas cualitativas basadas en el análisis del discurso vienen siendo instrumentos privilegiados del acercamiento a la infancia desde una posición epistemológica centrada en la población infantil y en particular los grupos de discusión y las entrevistas grupales en sus diferentes versiones, aderezadas con el uso de técnicas proyectivas como el uso de viñetas o historias inconclusas, se han revelado como técnicas de investigación particularmente útiles para conocer el mundo infantil (Gómez 2010, Rodríguez 2006, Urmeneta 2010).

En nuestro caso, hemos hecho uso de la entrevista grupal dentro de los propios contextos educativos. Se trataba de aprovechar nuestra presencia en los centros educativos para escoger un pequeño número de alumnado que respondiera a un sencillo perfil sociodemográfico y sentarnos con ellos durante al menos 45 minutos para discutir sobre los mismos aspectos que estaban en nuestro diseño conceptual, y acabaron entrando en el diseño del instrumento cuantitativo. Las entrevistas se celebraban por lo general en algún espacio del centro (nunca un aula) que previamente habíamos inspeccionado para comprobar que era idóneo (amplio, aislado del ruido del pasillo, sin obstáculos que impidieran la comunicación visual y preferiblemente dotado de una mesa de reuniones).

Un aspecto innovador que ha hecho diferir la dinámica de estas entrevistas de grupo de lo que hubiéramos diseñado para adultos ha sido la inclusión de pequeñas historias inconclusas a modo de viñetas discursivas que nos servían para lanzar los temas de los que queríamos discutir. Cuando alguno de los participantes en las entrevistas leía las historias propuestas solía iniciarse una discusión que ya no concluía hasta que nos veíamos obligados a apagar la grabadora porque la entrevista había acabado. Dos ejemplos del tipo de historias que hemos utilizado se muestran en el anexo II de este mismo texto.

Aunque en general el funcionamiento de estas entrevistas de grupo ha sido muy satisfactorio, hemos encontrado también algunas limitaciones, muchas de ellas derivadas del contexto en el que se realizaron o de elementos circunstanciales al propio trabajo de campo. En este sentido:

- Funcionaron mejor las entrevistas en las que los docentes no habían preseleccionado a los/as alumnos/as participantes. En ocasiones los docentes insistían en señalar a algunos estudiantes en concreto porque los consideraban "ejemplares" en un sentido o en otro. Sin embargo, sus criterios pueden ser poco relevantes 
en el contexto de una investigación, especialmente si se desconoce la dinámica de funcionamiento de una entrevista grupal.

- También dieron un mejor resultado las entrevistas en las que no había más de 4 o 5 alumnos. Grupos más grandes tienden a inhibir el discurso de algunos participantes y fácilmente producen una acalorada discusión en la que el discurso se hace ininteligible.

- La presencia de un adulto ajeno a la investigación tenía un fuerte efecto inhibidor sobre el discurso infantil. En raras ocasiones, algún docente entraba momentáneamente en la sala donde celebrábamos la entrevista, provocando el silencio del grupo.

- Dado que nos habíamos fijado el tiempo de desarrollo normal de una clase para estas entrevistas, hubo algunas en las que tuvimos que finalizar antes de que el grupo agotara los temas de discusión. Sin embargo, ésta era una condición necesaria para no interrumpir en exceso la vida académica de los participantes y no irritar al personal de los centros educativos.

\section{Resultados}

Desde un principio, el análisis de los datos correspondientes al estudio Infancia 2.0 (7) reflejó la singular complejidad del escenario digital tal y como es descrito por los propios menores de edad, particularmente en lo que se refiere al ámbito relacional con la población adulta. Hemos tenido presente siempre en nuestro análisis esta complejidad al hablar de los distintos conjuntos de fenómenos que dan forma a nuestra representación de la realidad, dado que nos interesa especialmente la visión infantil sobre su papel como sujetos con derechos dentro de este marco que es el nuevo escenario digital de la sociedad de la información.

\subsection{El riesgo como experiencia digital}

La cuestión del riesgo ha sido un aspecto importante durante la investigación, ya que nos daba pie a comentar con los entrevistados cuál era su papel ante determinados sucesos de este escenario de la comunicación digital. Los propios sujetos aludían a hechos y situaciones que fácilmente entran a formar parte de las peores experiencias del mundo virtual de los nuevos medios. Tengamos en cuenta, por ejemplo, que según nuestra investigación algo más de un $14 \%$ y un $8 \%$ de estos niños y niñas han visitado páginas web para adultos o han sido contactados por un desconocido en la red (8), respectivamente. Los propios niños y niñas andaluces, ante fenómenos tan novedosos como las redes sociales, se muestran alerta de sus riesgos: más de 41 de cada 100 entrevistados declaró conocer que las redes sociales pueden ser una amenaza. Lo curioso, y lo más interesante desde nuestra perspectiva, es que esta conciencia del riesgo no conduce a los participantes en nuestra investigación directamente a una posición de vulnerabilidad percibida ni a un recurso generalizado a figuras adultas a las que se demanda protección. En realidad, nos parece a la luz de los datos que la cuestión del riesgo aparece también hilada con la propia autonomía de estos menores de edad y su capacidad para poner en marcha soluciones concretas que ponen fin a diversas situaciones peligrosas o desagradables, lo que algunos autores identifican como una forma de resiliencia (Livingstone y Haddon, 2009). El siguiente fragmento de discurso ilustra bien esta discusión entre riesgos y respuestas ante los mismos o formas de sortearlos (entre paréntesis la edad del informante).

Niña (14): Yo me pongo muy nerviosa porque no sé quién es y normalmente esa persona es muy pesado, y siempre quiere saber algo más de ti y tú no le quieres decir nada y él sigue, él o ella.

Niño (14): Pues yo lo borro y ya está...

Niña (14): Y lo bloqueas y se hace otra cuenta en Tuenti y sigue abusándote.

Niño (14): No porque si tú no aceptas la cuenta, pues ya está.

Al mismo tiempo, ilustra otra cuestión que suele resultar invisible cuando los niños no son los informantes principales de una investigación: la importancia del apoyo producido por los pares. Tal y como hallamos en el transcurso de nuestro trabajo, algo más de una quinta parte $(21,1 \%)$ de todos los niños y niñas consultados afirmaban "haber dado consejos prácticos a amigos o conocidos sobre cómo proteger su 
identidad o evitar que les pase algo desagradable en internet". Es decir, estas estrategias de evitación del riesgo son, además, gestadas en el seno de una cultura de pares y difundidas horizontalmente a lo largo de la misma. La cuestión de la privacidad, por ejemplo, es muy ilustrativa de lo que queremos decir al afirmar que la relación entre lo adulto y lo infantil es compleja y, en ocasiones, conflictiva.

\subsection{Negociando con la persona adulta: la cuestión de la privacidad}

No cabe duda de que, en el conjunto de la representación infantil que estamos analizando, desempeña un papel fundamental la cuestión de la privacidad (uno de los derechos fundamentales señalados en la propia CDN). Ésta es tan importante por constituir una manifestación clara de nuestra identidad en tanto individuos separados de la masa de nuestros semejantes. Resulta, igualmente, un punto de fricción entre el imaginario adulto y el infantil, ya que lo que los niños y niñas ganan en autonomía solo puede ser producto de la pérdida de control directo de los adultos. Esto queda ejemplificado fácilmente por la lucha alrededor de las contraseñas. En el mundo digital, donde se vierte nuestra vida en soportes y aplicaciones muy diferentes, la contraseña es una débil frontera que nos separa de la mirada ubicua de los demás. Los niños, en este sentido, tienen una posición especialmente delicada: a los problemas conocidos por todos (extravío y olvido, también robo o "crackeo" de contraseñas de cuentas de correo electrónico y otros servicios, por citar sólo algunos ejemplos) suman la necesidad de sustraer (o no) éstas del control adulto, si quieren disfrutar de esa sensación de privacidad. Un dato puede sorprender a muchas personas adultas: casi el $72 \%$ de todos los niños y niñas consultados en Andalucía piensa que la gente de su edad "tiene derecho a poder usar contraseñas para que otras personas no lean su correo electrónico o sus documentos". La solución, en muchos casos, pasa por la negociación con el adulto sobre los propios límites de la privacidad, tal y como ilustra el discurso de esta niña:

\section{Entrevistadora: ¿tu madre tiene tu contraseña?}

Niña (14): Sí, me obligó a que se la diese. Y yo no la he podido cambiar, porque me dijo que si algún día me pasaba algo y la policía le pedía algo, ella tendría que enseñarlo y entonces se la tuve que dar. Pero ella no se mete, ella me dio la palabra de que ella no se iba a meter, entonces yo se la di. Pero si mi amigo me escribe algo privado, ella no tiene por qué enterarse.

Precisamente, el derecho a la privacidad y a la comunicación libre de miradas adultas (como expresaba este último informante: si me escribe algo privado, ella no tiene por qué enterarse), entendido particularmente como el derecho a proteger ésta a través de contraseñas en mano de los propios niños y niñas, se ha revelado como una de las cuestiones claves en el transcurso de esta investigación. En el siguiente apartado, en el que analizamos la manera en que niños y niñas perciben sus derechos como individuos en los nuevos entornos de la comunicación digital, aportamos una información más detallada a este respecto.

\subsection{Niños, niñas y derechos en el escenario de la cultura digital}

Vayamos directos a la cuestión de los derechos y su representación por parte de la población infantil andaluza, que ejemplifica bien el tipo de respuesta compleja que los sujetos aportan al debate sobre su posición en el nuevo mundo de la cultura digital. Para ello hemos analizado cuatro indicadores clave sobre la representación que estos niños y niñas sostienen a propósito de sus derechos y el papel de las personas adultas en el mundo de la comunicación digital. Estos indicadores se componen de: a) un ítem que mide el derecho al acceso a estas nuevas tecnologías por parte de la población infantil ("Los chicos/as de mi edad deberían poder tener un ordenador y acceso a internet"), b) otro ítem referido al derecho a la privacidad ("La gente de mi edad tiene derecho a contraseñas para que otros no lean su correo o documentos") y c) otros dos ítems que dan cuenta tanto del derecho a recibir protección por parte de los adultos ("Los chicos y chicas de mi edad tienen derecho a que las personas mayores les protejan de las cosas que no les gustan de internet") como de recibir asesoramiento y apoyo ("tiene derecho a que padres y profesores le ayuden y enseñen a utilizar mejor internet") (9). Se ha medido la respuesta con una escala que oscila entre un mínimo nivel de acuerdo expresado con la frase "no me gusta nada" (valor 1) a otro de máximo acuerdo o "me gusta mucho" (valor 4). Una media más elevada denotará, por tanto, un mayor nivel de acuerdo y al contrario (la pregunta en su redacción original puede encontrarse en el Anexo I de este texto). La tabla siguiente resume la información correspondiente a los cuatro indicadores, aportando igualmente un desglose de sus valores tanto en función del sexo como de la edad de los entrevistados. 


\begin{tabular}{|l|c|c|c|c|c|c|}
\hline $\begin{array}{l}\text { Los/as chicos/as de mi } \\
\text { edad... }\end{array}$ & $\begin{array}{c}\text { (\%) } \\
\text { escogieron } \\
\text { "me gusta } \\
\text { mucho" }\end{array}$ & $\begin{array}{l}\text { Media } \\
(1-4)\end{array}$ & $\begin{array}{l}\text { Media } \\
\text { Niños }\end{array}$ & $\begin{array}{l}\text { Media } \\
\text { Niñas }\end{array}$ & $\begin{array}{l}\text { Media } \\
<14\end{array}$ & $\begin{array}{l}\text { Media } \\
>14\end{array}$ \\
\hline $\begin{array}{l}\text { tienen derecho a que padres/ } \\
\text { profesores ayuden y enseñen } \\
\text { a utilizar mejor intemet }\end{array}$ & 33,90 & 2,95 & 2,80 & 3,09 & 3,00 & 2,92 \\
\hline $\begin{array}{l}\text { tienen derecho a que los } \\
\text { mayores les protejan de lo } \\
\text { que no les gusta de internet }\end{array}$ & 32,10 & 2,99 & 2,76 & 3,19 & 3,03 & 2,96 \\
\hline $\begin{array}{l}\text { deberían poder tener un } \\
\text { ordenador y acceso a internet }\end{array}$ & 66,20 & 3,58 & 3,56 & 3,60 & 3,54 & 3,60 \\
\hline $\begin{array}{l}\text { tienen derecho a contraseñas } \\
\text { para que otros no lean su } \\
\text { correo o documentos }\end{array}$ & 78,70 & 3,69 & 3,56 & 3,81 & 3,59 & 3,75 \\
\hline
\end{tabular}

Tabla 1. Grado de acuerdo sobre derechos e internet según sexo y edad Fuente: Proyecto Infancia 2.0 (2011)

Los cuatro ítems reúnen un grado de acuerdo significativo, siendo sin embargo éste claramente mayoritario en los dos últimos (derecho al acceso y a la privacidad). Al mismo tiempo la información tabulada revela una aceptación diversa de los cuatro enunciados propuestos afectada, tanto por la edad de los sujetos entrevistados como por su sexo. Naturalmente, para profundizar en el análisis y asegurarnos de que estas diferencias no son producto del azar, hemos recurrido a la prueba ANOVA de un factor. Después de calcular las medias generales de cada ítem, se ha efectuado un contraste estadístico partiendo primero de la comparación de medias en función del sexo de los entrevistados (dos niveles: niños y niñas) y después haciendo lo propio respecto a su edad (dos niveles: menores y mayores de 14 años). La operación ha deparado algunos resultados muy interesantes. Por ejemplo, encontramos un efecto de género en tres de los cuatro ítems analizados. Así, las diferencias de medias observadas en el contraste del primer ítem, referido al derecho a recibir ayuda, fue de $F(1,773)=18,35, p<0,001$; en el segundo ítem, relativo a la protección por parte de los mayores, se obtuvo una $F(1,769)=42,64, p<0,001$ y en el cuarto ítem, relacionado con el derecho a disponer de contraseñas, el resultado fue de $F(1,770)=23,39, p<0,01$. En estas tres cuestiones las niñas mostraron puntuaciones significativamente más altas que los niños. El ítem $n^{\circ} 3$ referido al derecho al acceso a internet no parece arrojar diferencias significativas entre niños y niñas $(p=0,49)$. En lo que toca a la edad, los resultados son estadísticamente menos significativos, pero igualmente interesantes. En este caso, el único ítem en el que se encuentran diferencias estadísticamente significativas es el referido al acuerdo respecto al derecho a disponer de contraseñas. Conforme aumenta la edad, se incrementa la media de acuerdo respecto al derecho a disponer de contraseñas para proteger la privacidad (ítem $\left.n^{\circ} 4 ; F(1,770)=8,55, p<0,005\right)$. Niños y niñas más mayores, por tanto, parecen ser también personas más concienciadas sobre el derecho a su privacidad.

Todos estos datos tienen una clara coherencia interpretativa y abundan en una imagen compleja de la posición de los y las menores de edad en relación a las personas adultas en el mundo de los nuevos medios de información y comunicación. Es obvio que existe un consenso valorativo muy alto en torno a dos cuestiones básicas: el derecho al acceso a los ordenadores e internet y el derecho a disponer de contraseñas que protejan la privacidad de los usuarios (este último ítem es el que recibe más apoyo por los y las encuestados/as). Existe un consenso también destacable, aunque de menor magnitud, en torno al derecho tanto a recibir protección por parte de las personas adultas como a ser acompañados y guiados por ellas (más de un tercio de los niños y niñas en Andalucía apoyan estas afirmaciones). Este último dato es rico y admite múltiples interpretaciones, ya que tiene una relación con la propia autonomía percibida por 
parte de los usuarios. Precisamente, al detallar los resultados en función de otras variables hemos podido comprobar cómo la edad introduce un sesgo claramente dirigido a incrementar la necesidad de disponer de la propia privacidad; por contra, desde una interpretación basada en el género, podemos destacar cómo el rechazo a la protección adulta es mucho más acusado entre los chicos, particularmente los mayores de 14 años, mientras que las chicas muestran una preferencia más clara a disponer de figuras adultas cercanas que brinden tanto protección como apoyo y formación, si bien acentúan igualmente su derecho a la privacidad.

Por tanto, la representación que niños y niñas hacen de sus derechos en relación a la población adulta vuelve a estar cargada de cierta ambivalencia que identifica a estos últimos tanto como garante de esos derechos y fuente de ayuda y protección, como amenaza potencial para la autonomía infantil y para esos mismos derechos. Precisamente, al triangular la información obtenida tanto por instrumentos cuantitativos como cualitativos, hemos detectado la presencia de esta misma "representación ambivalente" también en el discurso infantil. El siguiente fragmento ilustra bien lo paradójico del papel representado por los adultos en muchas situaciones cotidianas, al tiempo que es muestra de un discurso centrado, sobre todo, en el respeto a la privacidad de las personas menores de edad:

Niña (14): Lo que pasa es que nosotras teníamos una guardia, no había un profesor, entonces vino otro a cubrir, y traía un ordenador y nos dejó conectarnos. Entonces estábamos las dos en Tuenti y vio a un compañero y nos dijo que se la enseñáramos. Y nosotras le dijimos que no, que no teníamos que enseñarle todo a los profesores y se lo tuvimos que dar.

\section{Discusión y conclusiones}

El recurso a niñas y niños como informantes suele deparar una imagen compleja y rica de la población menor de edad y sus intereses. Esta investigación, a tenor de los datos manejados y expuestos en este texto, no es una excepción. Ni las etiquetas aparentemente afortunadas, como "nativo digital", ni la aproximación mecanicista que supone que las nuevas tecnologías de la información y comunicación simplemente "impactan" por igual sobre los menores de edad sin que estos jueguen un papel destacable en su apropiación, parecen explicar convincentemente esta complejidad. Los datos y referencias que hemos manejado sostienen, más bien, la impresión de que el proceso de apropiación de estos nuevos medios de comunicación es todavía más indeterminado de lo que parece y resulta de una apropiación cultural e interpersonal compleja dentro del seno de la cultura infantil (De Almeida y otros 2011, Núñez-Gómez 2012). Apropiación que incluye desde edades bien tempranas una concepción, por más que sea básica, de sus derechos como individuos, así como una representación de su propia condición como usuarios expuestos al riesgo que está muy lejos de basarse en su vulnerabilidad o inmadurez y que, además, coloca a los adultos en una situación paradójica.

La ambivalencia parece ser una de las constantes de esta percepción que, desde la mirada de niños y niñas, trata de desentrañar el nuevo mundo de lo digital. Los datos cuantitativos y cualitativos así lo atestiguan y está presente en el discurso infantil: riesgo y potencialidades aparecen juntos, como ejemplo más claro de un patrón que se repite insistentemente. Los niños y niñas se refieren a las fuentes de riesgo en la misma medida en que parecen confiar en sus propios recursos para afrontarlo. Aparece también, dentro de su representación la posibilidad de producir un apoyo grupal que circula dentro del círculo de pares (consejos, asesoramiento, etc.). Esta percepción de sí mismos como usuarios autónomos, no necesitados de una especial protección ni control es mucho más acusada entre los chicos que entre las chicas y en algunos aspectos, como la protección de su privacidad y de las comunicaciones con sus pares, parece crecer con la edad. Las niñas, en cambio, se representan en mayor medida como usuarias con derecho a ser asesoradas y protegidas, aunque son igualmente más celosas en la protección de su privacidad frente a intromisiones adultas.

Sin embargo, esta autonomía, tal y como es representada por la población infantil, no se fundamenta en un rechazo frontal a lo adulto, sino colocando a este en una posición de cierto extrañamiento respecto de la vida infantil que, sin embargo, no renuncia a la proximidad. Se detecta frecuentemente en el discurso infantil una doble representación de las figuras adultas: como figuras de control dotadas de presencia un tanto "invasiva", que sobredetermina su papel de vigilante y protector por encima de ciertos límites que la población infantil considera importantes; pero también como fuente de apoyo y protección necesaria. Las 
niñas y niños parecen estar construyendo así una representación en la que el adulto debe situarse como una figura de apoyo distante, presente en el imaginario infantil y dispuesto a proporcionar tanto el apoyo como la protección, pero siempre respetuosa con el espacio de autonomía y privacidad que demandan para sí.

No es de extrañar, por tanto, que el más alto consenso hallado por esta investigación en torno a la percepción que los niños y niñas andaluces tienen de sus derechos en el escenario de los nuevos medios digitales sea el del derecho a la privacidad. Chicas y chicos desean que existan fronteras al control adulto, si bien apuntan a que estas pueden ser negociadas. Casi con la misma intensidad los niños entienden que el acceso a internet y a la comunicación digital debe ser algo de lo que pueda disfrutar toda la población infantil. Aunque el consenso sea menor en otros aspectos, puede colegirse de los datos expuestos hasta ahora que la representación infantil de sus derechos es compleja y viene a sostener las impresiones vertidas en párrafos anteriores. Existe una percepción clara del derecho a ser protegido y ayudado por los adultos, por ejemplo, pero reúne un menor consenso valorativo al ser también una fuente potencial de irrupciones en el disfrute autónomo de estos nuevos medios, particularmente en lo que se refiere a la protección de la privacidad y de las comunicaciones que circulan dentro del grupo de pares.

A la luz de estos hallazgos, no cabe duda de que la población infantil demanda una aproximación compleja a nuestro papel como adultos en un escenario digital en el que riesgo y potencialidades beneficiosas se dan la mano y, en muchos casos, resulta incluso difícil determinar si hablamos de una cosa o de la otra. Estamos de acuerdo con Livingstone y Haddon (2009: 22) cuando afirman que el discurso sobre los riesgos y oportunidades de los nuevos medios esconde una cuestión referida a los derechos de la población infantil. En este contexto, en el que probablemente encontraremos muchas situaciones en las que distintos derechos entren en conflicto (libertad de expresión vs. libre acceso a la información o protección de la infancia, por ejemplo), sólo una mirada respetuosa y basada verdaderamente en un enfoque de derechos puede garantizar que no producimos un diagnóstico sesgado sobre la base de la subestimación tanto de la capacidad y autonomía de la población infantil como de la complejidad de la vida social de niños y niñas en el mundo incipiente de la sociedad de la información.

\section{Notas}

1. Este artículo fue producido gracias a la financiación y apoyo de la Fundación Centro de Estudios Andaluces a través del proyecto Infancia 2.0: redes sociales y usos tecnológicos emergentes en la población nativa digital andaluza (referencia PR022/10). Dicho proyecto fue aprobado en la correspondiente convocatoria 2010 de ayudas a proyectos de investigación. Igualmente, los autores agradecen a la Universidad de Huelva y al grupo de investigación Estudios Sociales e Intervención Social (eseis.es) el apoyo prestado en forma material y logística a la hora de ejercer nuestra labor como investigadores.

2. Lamentablemente, el editor español del libro nos ha hurtado la referencia a la muerte de la infancia (the death of childhood) del título original, titulándola simplemente: crecer en la era de los medios electrónicos.

3. De acuerdo con los datos proporcionados por Eurostat en 2012 (acceso 10-01-2013): http://epp.eurostat.ec.europa.eu/portal/page/portal/product_details/publication?p_product_code=KS-SF-12$\underline{050}$

4. Nuestro análisis guarda en realidad un sentido de género y parte de la idea de que niños y niñas asimilan a la vez que construyen papeles sociales en torno a sus roles diferenciados. Sin embargo, en este estudio, como se verá, utilizamos una única variable nominal dicotómica alrededor de los valores "niño" y "niña". Por esta razón preferimos denominarla "sexo" siempre que nos estemos refiriendo, concretamente, a nuestra explotación de datos y, en todo caso, nos referimos al género al hacer una interpretación de los mismos.

5. El término infancia es fuertemente polisémico y alrededor de él se ha desarrollado una enrevesada nomenclatura de etiquetas que se solapan y en muchos casos albergan connotaciones discutibles. En este texto, dado que en nuestra investigación trabajamos con un rango de edad amplio, hemos evitado el habitual enredo de distinguir la infancia de la adolescencia, la preadolescencia y la adolescencia, así como hemos 
prescindido de la muy estigmatizadora etiqueta de "menores", tan frecuente en el español coloquial. En su lugar nos ceñimos al criterio que marca la Convención de Derechos del Niño, que considera niño a toda persona menor de edad. Por tanto, nos referiremos indistintamente en nuestro análisis a niños y niñas, población infantil o personas menores de edad.

6. Para el análisis de los datos cuantitativos hemos usado el paquete estadístico SPSS en su versión 15. En lo que concierne al análisis cualitativo nos hemos apoyado en el programa Atlas.Ti en su versión 6.0.

7. Más información sobre el proyecto y sus principales resultados puede encontrarse en Rodríguez, Gualda y otros 2011.

8. Algo detectado comúnmente en otros estudios referidos a la realidad digital de la infancia y juventud española, véase por ejemplo el texto reciente de Cáceres 2013.

9. Se observará que, en realidad, estos cuatro ítems evocan dos de los tres principios fundamentales de la Convención de Derechos del Niño: Provisión (acceso) y Protección, teniendo igualmente el derecho a la privacidad su reflejo en el articulado de la CDN y siendo, por otro lado, prerrequisito obvio para el ejercicio del tercer principio: la Participación.

\section{Bibliografía}

Amich-Elías, Cristina

2007 "Derecho a la protección de la infancia versus libertad de expresión en internet: Unión Europea y Brasil", Derechos y Libertades, 17, Época Il: 171-198.

Area, Manuel (y Teresa Pessoa)

2012 "De lo sólido a lo líquido: las nuevas alfabetizaciones ante los cambios culturales de la Web 2.0.", Comunicar, $\mathrm{n}^{\circ} 38$ (19): 13-20.

Buckingham, David

2000 After the Death of Childhood. Growing Up in the Age of Electronic Media. Londres, Polity Press.

Cabrera García, Jossue

2013 "Perspectivas teóricas de Cibercultura, y su aplicación en espacios educativos", Gazeta de Antropología, 29 (1), artículo 08 .

http://hdl.handle.net/10481/26479

Cáceres Zapatero,María Dolores (y otros)

2013 "Comunicación interpersonal en la web 2.0. Las relaciones de los jóvenes con desconocidos", Revista Latina de Comunicación Social, nº 68: 436- 456.

Colorado Castellary, Arturo

2010 "Perspectivas de la cultura digital", ZER Revista de Estudios de Comunicación, n² 28: 103-115.

http://www.ehu.es/zer/es/hemeroteca/articulo/perspectivas-de-la-cultura-digital/421

Dean, Jodi

1999 “Virtual Fears”, Signs (24) 4: 1169-1078.

De Almeida, Ana Nunes (y otros)

2011 "Children and digital diversity: From 'unguided rookies' to 'self-reliant cybernauts", Childhood, no 19 (2): 219-234.

García García, Francisco (y María J. Rosado Millán)

2012 "Conductas sociocomunicativas de los nativos digitales y los jóvenes en la web 2.0", Communication 
and Society/Comunicación y Sociedad, vol. XXV (1): 15-38.

Garde, Amandine

2011 "Advertising regulation and the protection of children-consumers in the European Union: in the best interest of... commercial operators?", International journal of children's rights, $\mathrm{n}^{\circ} 19$ (3): 523-545.

2012 "The best Interest of the Child and EU Consumer Law and Policy: A Major Gap between Theory and Practice?", en James Devenny and Mel Kenny (eds.), European Consumer Protection: Theory and Practice. Cambridge University Press, Cambridge: 164-201.

Gómez Espino, Juan Miguel

2012 “El grupo focal y el uso de viñetas visuales en la investigación con niños”, Empiria, n² 24: 45-66.

Hirsjärvi, Irma (y Sami Tayie)

2011 "Niños y nuevos medios: estudios de caso en Egipto y en Finlandia", Comunicar, n 37 (19): 99-108.

Hodson, Katherine

2010 "Moral Panics over Contemporary Children and Youth", The International Journal of Children's Rights, n० 18 (1): $149-153$

Kuipers, Giselinde

2006 "The social construction of digital danger: debating, defusing and inflating the moral dangers of online humor and pornography in the Netherlands and the United States", NewMedia \& Society, n 8: 379-400.

Livingstone, Sonia (y Leslie Haddon)

2009 EU Kids Online: Final report. London School of Economics. London: EU Kids Online.

http://www.eukidsonline.net

Naciones Unidas

1989 Convención de Derechos del Niño. Nueva York, Naciones Unidas.

Núñez-Gómez, Patricia (y otros)

2012: "Tendencias de las relaciones sociales e interpersonales de los nativos digitales", Revista Latina de Comunicación Social, $n^{\circ}$ 67: 179-206.

O'Neill, Brian

2010 "Media Literacy and Communication Rights: Ethical Individualism in the New Media Environment", International Communication Gazette, $\mathrm{n}^{\circ} 72$ (4-5): 323-338.

Prensky, Marc

2001 "Digital natives, digital immigrants", On the Horizon, n 9 (5): 1-6.

Rodríguez-Pascual, Iván

2006 "Redefiniendo el trabajo metodológico cualitativo con niños: el uso de la entrevista de grupo aplicada al estudio de la tecnología", Empiria, no 12: 65-88

2010a "Entre la protección y la participación: sujetos y discursos en el marco normativo sobre la infancia y las nuevas tecnologías de la información en la UE”, Comunicación presentada en el $X$ Congreso español de Sociología. Pamplona.

2010b "E-Generaciones: ¿Cuánto hay de Adultocéntrico en el Análisis de la Relación entre la Población Infantil y las Nuevas Tecnologías?", Psychosocial Intervention -Intervención psicosocial, nº 19 (1): 9-18.

Rodríguez-Pascual, Iván (y otros)

2011 "La población infantil ante las nuevas tecnologías de la información: una aproximación a la realidad de los nativos digitales andaluces", Actualidad, $n^{\circ}$ 63. Sevilla, Fundación Pública Centro de Estudios Andaluces.

Ruxton, Sandy

2005 Children's Rights in the EU: Next Steps.

http://www.europeanchildrensnetwork.org/. 
Selwyn, Neil

2003 "Doing It for the Kids': Re-examining Children, Computers and the Information Society", Media Culture \& Society, $\mathrm{n}^{\circ} 25:$ 351-378.

Savirimuthu, Joseph

2011 "The EU, Online Child Safety and Media Literacy", The International Journal of Children's Rights, n 19 (3): 547-569

Urmeneta Garrido, Ana R.

2010 "El Cuento Inconcluso. Una herramienta sociológica innovadora para el estudio del mundo social infantil", X Congreso FES-Pamplona.

Vergara, Enrique (y Ana Vergara)

2012 "Representación de la infancia en el discurso publicitario en Chile”, Comunicar, $\mathrm{n}^{\circ} 38$ (19): 167-174.

Wold, Thomas

2010 "Protection and access: To regulate young people's internet use", International Journal of Media and Cultural Politics (6) 1: 63-80.

\section{ANEXO I. Fragmento del cuestionario: redacción completa de la pregunta $n^{\circ} 17$}

Ahora te proponemos una serie de frases y queremos que nos indiques hasta qué punto te gustan (estás de acuerdo) o no te gustan (no estás de acuerdo). (Marca una sola cruz por cada frase).

\begin{tabular}{|c|c|c|c|c|}
\hline & $\begin{array}{l}\text { 1. No } \\
\text { me } \\
\text { gusta } \\
\text { nada }\end{array}$ & $\begin{array}{l}\text { 2. No } \\
\text { me } \\
\text { gusta }\end{array}$ & $\begin{array}{l}\text { 3. Me } \\
\text { gusta } \\
\text { un } \\
\text { poco }\end{array}$ & $\begin{array}{c}\text { 4. Me } \\
\text { gusta } \\
\text { mucho }\end{array}$ \\
\hline $\begin{array}{l}\text { a) Internet puede ser útil para mí para los chicos y } \\
\text { chicas que conozco. }\end{array}$ & & & & \\
\hline $\begin{array}{l}\text { b) Es bueno que chicos y chicas como yo podamos } \\
\text { navegar solos por la red y buscar la información que } \\
\text { queremos. }\end{array}$ & & & & \\
\hline $\begin{array}{l}\text { c) Pienso que tengo derecho a que mis padres y mis } \\
\text { profesores me ayuden y me enseñen a utilizar mejor } \\
\text { Internet. }\end{array}$ & & & & \\
\hline $\begin{array}{l}\text { d) Creo que los chicos y chicas de mi edad tenemos } \\
\text { derecho a que las personas mayores que nosotros nos } \\
\text { protejan de las cosas que no nos gustan de Internet. }\end{array}$ & & & & \\
\hline $\begin{array}{l}\text { e) Todos los chicos y chicas de mi edad deberían } \\
\text { poder tener un ordenador y acceso a Internet para } \\
\text { conectarse. }\end{array}$ & & & & \\
\hline $\begin{array}{l}\text { f) La gente de mi edad tiene derecho a poder usar } \\
\text { contraseñas para que otras personas no lean su correo } \\
\text { electrónico o sus documentos. }\end{array}$ & & & & \\
\hline
\end{tabular}




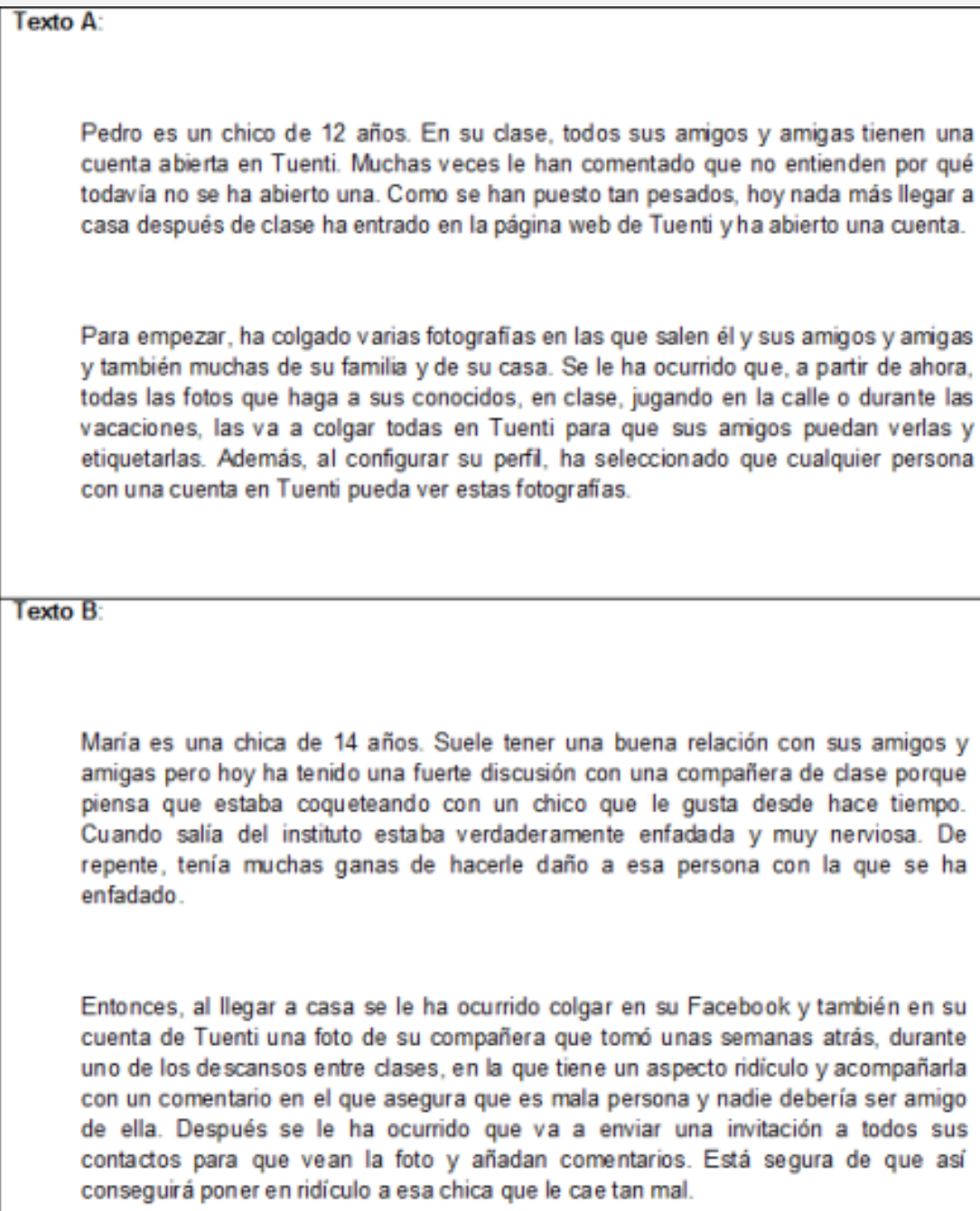

Pedro es un chico de 12 años. En su clase, todos sus amigos y amigas tienen una cuenta abierta en Tuenti. Muchas veces le han comentado que no entienden por qué todavia no se ha abierto una. Como se han puesto tan pesados, hoy nada más llegar a casa después de clase ha entrado en la página web de Tuenti y ha abierto una cuenta.

Para empezar, ha colgado varias fotografias en las que salen él y sus amigos y amigas y también muchas de su familia y de su casa. Se le ha ocurrido que, a partir de ahora, todas las fotos que haga a sus conocidos, en clase, jugando en la calle o durante las vacaciones, las va a colgar todas en Tuenti para que sus amigos puedan verlas y etiquetarlas. Además, al configurar su perfi, ha seleccionado que cualquier persona con una cuenta en Tuenti pueda ver estas fotografias

Texto B:

Maria es una chica de 14 años. Suele tener una buena relación con sus amigos y amigas pero hoy ha tenido una fuerte discusión con una compañera de clase porque piensa que estaba coqueteando con un chico que le gusta desde hace tiempo. Cuando salia del instituto estaba verdaderamente enfadada y muy nerviosa. De repente, tenía muchas ganas de hacerle daño a esa persona con la que se ha enfadado.

Entonces, al llegar a casa se le ha ocurrido colgar en su Facebook y también en su cuenta de Tuenti una foto de su compañera que tomó unas semanas atrás, durante uno de los descansos entre clases, en la que tiene un aspecto ridiculo y acompañarla con un comentario en el que asegura que es mala persona y nadie debería ser amigo de ella. Después se le ha ocurrido que va a enviar una invitación a todos sus contactos para que vean la foto y añadan comentarios. Está segura de que así conseguira poner en ridiculo a esa chica que le cae tan mal.

Cuadro 1. Dos ejemplos de materiales discursivos utilizados en las entrevistas grupales del proyecto Infancia 2.0. Fuente: Proyecto Infancia 2.0 (2011) 